\title{
Conductivity Recovery Around Point Electrodes in Electrical Geo-Tomography
}

\author{
A.D. Garnadi ${ }^{1}$, R.S. Anderssen ${ }^{2}$ \\ ${ }^{1}$ Dept. Mathematics, Institut Pertanian Bogor, \\ Jl. Meranti, Kampus IPB Darmaga, Bogor, 16680 Indonesia \\ ${ }^{2}$ CSIRO Mathematics, Informatics and Statistics, Canberra, Australia.
}

\begin{abstract}
We consider the inverse electrical Geo-Tomography problem, where the case of piecewise constan conductivities is mostly valid under the currents injection at adjacent point electrodes and the resulting voltages measured between the remaining electrodes.

The linear functional strategy from related problem in aquifer transmittivity is adapted to recover the conductivity constants in the vicinity zone containing the point electrodes. Such reconstruction method is needed as an initial step before full reconstruction of conductivity inside the domain from electrode measurements at the surface, as done in electrical GeoTomography.
\end{abstract}

\section{Introduction : Electrical GeoTomography as Parameter Identification Problem [7]}

In the study of a direct current geoelectric sounding, we modeled the phenomena within a bounded object $\Omega$, with the governing equation is the following elliptic partial differential equation:

$$
-\nabla(\sigma \nabla u)=q \quad \text { in } \quad \Omega
$$

where $\Omega \in R^{d},\{d=2,3\}$ is a bounded domain, $\sigma: \Omega \rightarrow R$ denotes the conductivity coefficient, $u: \Omega \rightarrow R$ is electrical potential, $q:=I\left(\delta\left(r-s_{i}\right)-\delta\left(r-s_{j}\right)\right)$ is current injection pair through point electrode at $s_{i, j}, i \neq j, 1 \leq i, j, \leq N$, where $N$ denoting the number of electrodes. When the current source term $q$ and the coefficient $\sigma$ is given, with an appropriate prescribed boundary information for solution $u$ so that the direct (forward) problem of $u$ is uniquely solvable in $\Omega$. Conversely, the recovery information about the coefficient of conductivity $\sigma$, given the pair $(q, g)$ is given, where $g\left(r_{k}\right):=u\left(r_{k}\right)$ 
is measured potential at point electrodes at $r_{k}$ due to current injection pattern $q$, is an inverse problem with $\sigma$ being sensitive due to perturbation in $u$ and $q$. The recovery of $\sigma$ from point electrodes measurements $u$ and $q$, utilizing the equation (1), that is Electrical GeoTomography with point electrode models.

\section{The Chow-Anderssen Ansatz [1],[2],[3],[4]}

The essentials ill-posedness in the recovery of information about the conductivity $\sigma$ from measurements of $u$ and $q$ relates to the fact that the observed electrical potential data $u$ must be differentiated. This is clear from the structure of equation (1). The essence of the ansatz involves four steps [1],[2] :

a. the reformulation of the partial differential equations model in terms of a weak form,

b. the application of integration by parts to move the differentiation of the observed potential onto the analytic test function,

c. the localization of the resulting linear functional representation for the conductivity to a subregion surrounding the point electrodes where the conductivity is assumed to be constant, and

d. the choice of the shape of the localization and the form of test function that facilitates the computational performance of the resulting algorithm.

As explained in Chow and Anderssen [4] in terms of the notation of equation (1) and the application of steps (a)-(d) of the ansatz, various linear functional formulas for the estimation of $\sigma$ on the subregion $\Lambda \subset \Omega$ can be derived which depends on the assumed regularity of the solution $u$ of equation (1) and the test function $v$.

One of the schemes to estimate $\sigma$ provided in [3] is used here, i.e.

$$
\hat{\sigma}=-\frac{\int_{\Lambda} q v d x}{\int_{\Lambda} u \cdot \Delta v d x}, \quad u \in L^{2}(\Lambda), \quad v \in H_{0}^{2}(\Lambda) .
$$

The special form that it take is a direct exploitation of the assumtion that $\sigma$ is a constant in the subregion $\Lambda$. In this scheme, through an integration by parts in conjunction with the regularity imposed on the test function $v$, the evaluation of $\hat{\sigma}$ does not involve a differentiation of $u$ since it has been transferred to $v$.

\section{The Recovery Formula [3]}

In practice, for given current injection $q:=I\left(\delta\left(r-s_{i}\right)-\delta\left(r-s_{j}\right)\right)$ is current injection pair through point electrode at $s_{i, j}$, let $r_{m}$ is measurement point position such that $r_{m} \subset \Lambda, \operatorname{supp}(q) \cap \Lambda \neq \emptyset$. If we choose $v$ is the solution of the following:

$$
\Delta v=\delta\left(r-r_{m}\right),\left.v\right|_{\partial \Lambda}=0, \quad v \in H^{2}(\Lambda) .
$$


Hence the scheme (2) resulted in:

$$
\begin{gathered}
\hat{\sigma}=-\frac{\int_{\Lambda} q v d x}{\int_{\Lambda} u \cdot \Delta v d x}=-\frac{\int_{\Lambda} q v d x}{u\left(r_{m}\right)} . \\
\hat{\sigma}=- \begin{cases}\frac{I\left(v\left(s_{i}\right)-v\left(s_{j}\right)\right)}{u\left(r_{m}\right)}, & s_{i}, s_{j} \in \Lambda, \\
\frac{I v\left(s_{i}\right)}{u\left(r_{m}\right)}, & s_{i} \in \Lambda, s_{j} \notin \Lambda, \\
\frac{-I v\left(s_{j}\right)}{u\left(r_{m}\right)}, & s_{i} \notin \Lambda, s_{j} \in \Lambda .\end{cases}
\end{gathered}
$$

62

Hence, we able to recover the conductivity arounds measurement point at $r_{m}$.

\section{${ }_{63}$ The Simulation}

64 We consider a rectangular domain $\Omega:=[-1,1] \times[-1,1]$, where we prescribed the fol65 lowing

1. $N_{e l}=16$, numbers of electrodes situated at $s_{i}=1, \cdots, 16$.

67

2. Current patterns: Wenner, i.e. $q:=I\left(\delta\left(r-s_{i}\right)-\delta\left(r-s_{i+3}\right)\right)$

68

The background conductivity $\sigma_{0}$ is 1.0 throughout the domain except an inclusion 69 which has conductivity $\sigma_{1}=50$. 


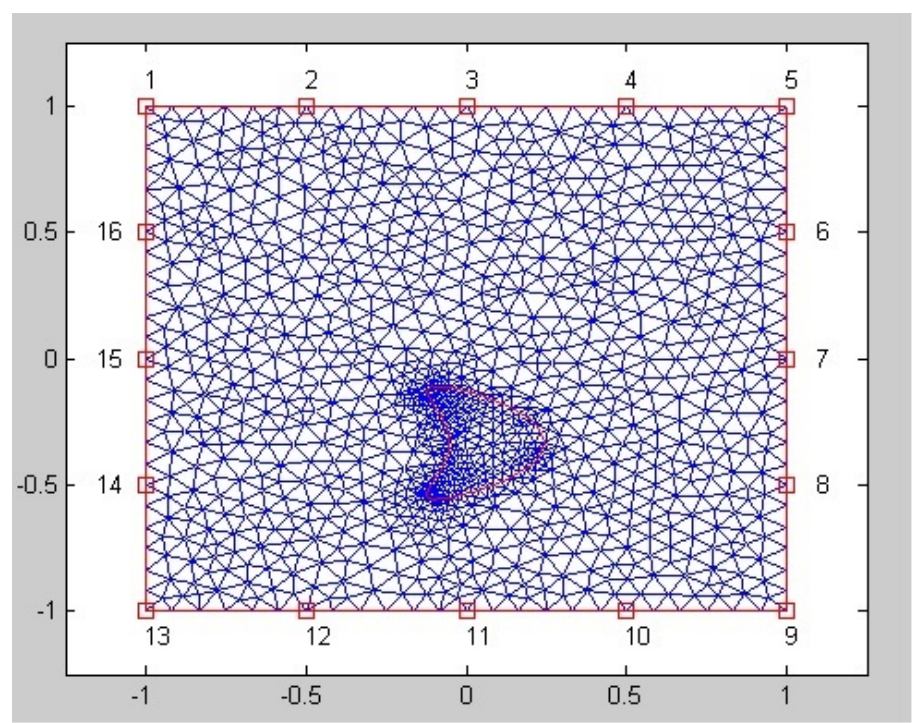

The data synthetically were generated using finite element methods which is parts of EIT reconstruction packages from [6].

Due to the numbers of electrodes are limited on each side $\Omega$ and to the choice of 75 adjacent current patterns in our numerical experiment, the type of regions for the test 76 function we use only two type of patches, due to symmetry. 


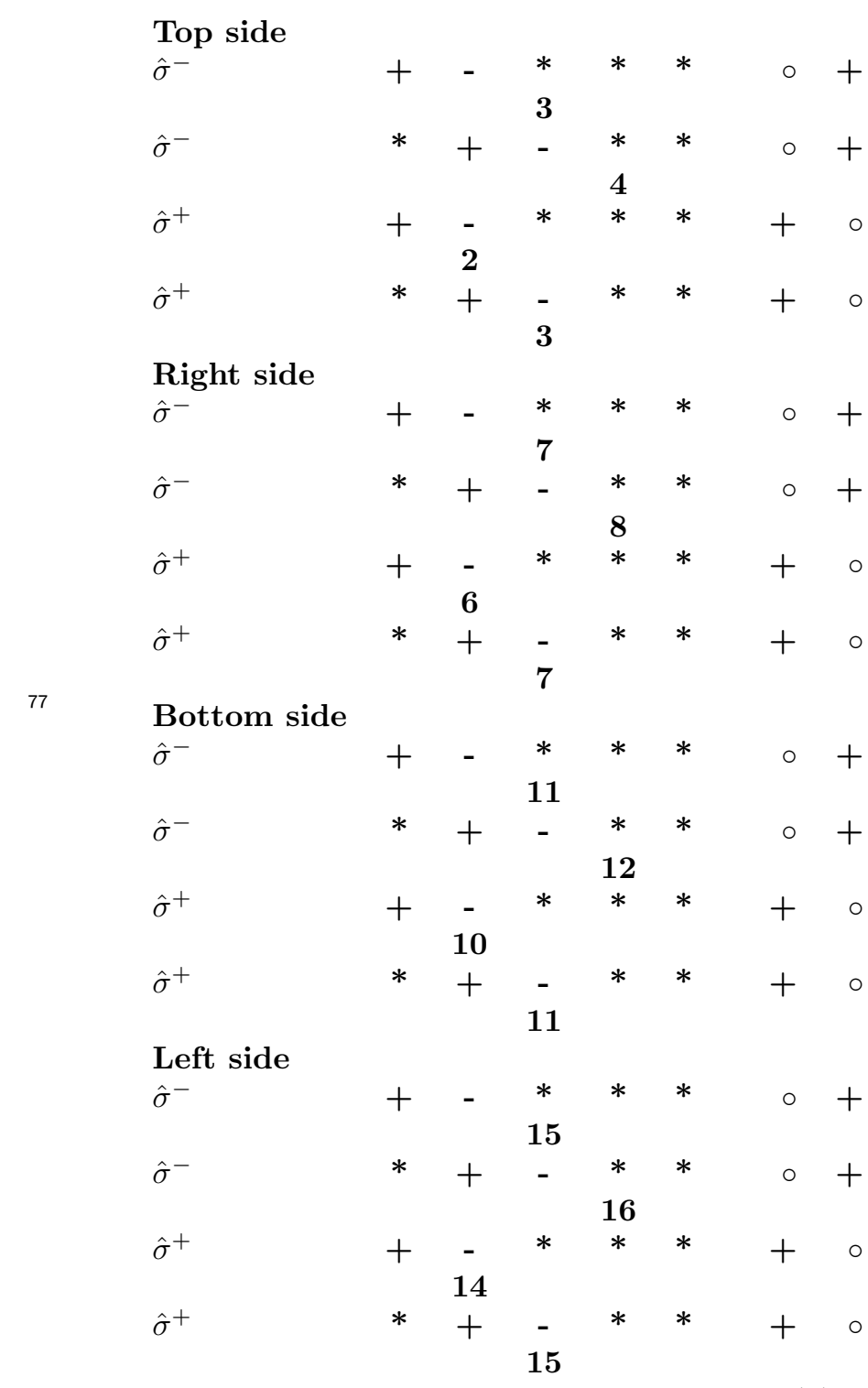

The test function $v$ is solving the equation (3):

$$
\Delta v=\delta\left(r-r_{m}\right),\left.v\right|_{\partial \Lambda}=0, \quad v \in H^{2}(\Lambda) .
$$

79 using Argyris element [5].

80

\section{Remarks}

- Note that the recovery formula also valid for general case of current patterns $q$ under point electrode models in EIT, furthermore the assumption that the problem is under homogeneous background can be ruled out. 
- In [4], an adaptive method was proposed, where adaptivity is worked out in shrinking zones manners. However, in the case of EIT with PEM, the techniques can be adapted in reverse by starting from a point expands into a larger constant zonation. This strategy can be exploited using probing function (3):

$$
\Delta v=\chi_{\Lambda},\left.v\right|_{\partial \Lambda}=0,
$$

where $\chi_{\Lambda}$ is characteristic function of $\Lambda$.

\section{Conclusions}

We propose a method to recover conductivity value in the vicinity of electrodes from the electrical Geo-Tomography problem, where the case of piecewise constan conductivities is mostly valid. The method adapted from the linear functional strategy from related problem in aquifer transmittivity to recover the conductivity constants in the vicinity zone containing the point electrodes.

\section{References}

[1] R.S. Anderssen \& S.S. Chow, 1991, Resolving the transmissivity zonation in confined aquifer, Mini Conference in Inverse problems, Proc. CMA Australian National University, pp 247-261.

[2] R.S. Anderssen \& C. Dietrich, 1987, The inverse problem of aquifer transmissivity identification, Research Report CMA-R01-87 Centre for Mathematical Analysis Australian National University, pp 247-261.

[3] R.S. Anderssen \& B.P. Lamichhane, 2011, Piecewise Constant Aquifer Parameter Identification Recovery, in MODSIM2011, 19th International Congress of Modelling and Simulation, pp 364-370.

[4] S.S. Chow \& R.S. Anderssen, 1991, Determination of the transmissivity zonation using a linear functional strategy, Inverse problems, 7(6), pp 841-851.

[5] Domínguez, Víctor and Sayas, Francisco-Javier, 2008, Algorithm 884: A simple Matlab implementation of the Argyris element, ACM Transactions on Mathematical Software (TOMS), Vol. 35 (2), 16

[6] A. Hellfrich-Scharbanenko \& T. Kreuzmann, 2008, MATLAB software guide for 2D Electrical Impedance Tomography,

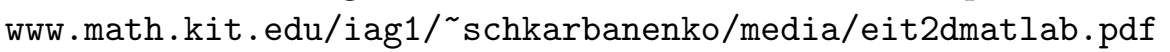

[7] A. Hellfrich-Scharbanenko, 2011, Elektrische Impedanztomografie in der Geoelektrik, Dissertation, Karlsurher Institut fuer Technologie. 